\title{
Significance of monocyte chemoattractant protein-1 in angiogenesis and survival in colorectal liver metastases
}

\author{
HIROYUKI YOSHIDOME $^{1 *}$, HIROHIKO KOHNO ${ }^{1,2^{*}}$, TAKASHI SHIDA ${ }^{1}$, FUMIO KIMURA ${ }^{1}$, \\ HIROAKI SHIMIZU $^{1}$, MASAYUKI OHTSUKA ${ }^{1}$, YUKIO NAKATANI ${ }^{2}$ and MASARU MIYAZAKI ${ }^{1}$
}

Departments of ${ }^{1}$ General Surgery and ${ }^{2}$ Diagnostic Pathology, Chiba University Graduate School of Medicine, Chiba, Japan

Received November 5, 2008; Accepted January 19, 2009

DOI: 10.3892/ijo_00000218

\begin{abstract}
Monocyte chemoattractant protein-1 (MCP-1) has been demonstrated to play a role in tumor progression. The present study examined the MCP-1 expression of colorectal liver metastases and determined whether MCP-1 is related to tumor progression and is a predictive marker for survival after hepatic resection of colorectal liver metastases. Eightyseven patients with colorectal liver metastases were evaluated by immunohistochemistry of MCP-1, Angiopoietin-2, CD68, and CD34 for determination of microvessel density. Clinicopatholgical data were also examined. In a separate experiment, immunohistochemistry of MCP-1 was performed to investigate the expression of primary colorectal tumor according to the clinical stage. MCP-1 mRNA expression was determined in colorectal cancer cell lines. Forty-nine patients $(56 \%)$ showed high expression of MCP-1 of colorectal liver metastases. High MCP-1 expression was related to multiple colorectal liver metastases. When the degree of MCP-1 expression increased, microvessel density count significantly increased compared with low MCP-1 expression. The MCP-1 expression correlated with Angiopoietin-2 expression. MCP-1 expression of the primary colorectal cancer increased as the clinical stage advanced. The increased MCP-1 mRNA expression was observed in cancer cell lines which have high metastasis potency. Univariate analysis demonstrated that the timing of metastases, tumor size, number of metastases, and MCP-1 expression were significant prognostic factors. Multivariate analysis demonstrated that MCP-1 expression was a significant prognostic factor in hepatic disease-free survival. The MCP-1 expression in colorectal liver metastases, at least in part, may be associated with angiogenesis and be a predictive marker
\end{abstract}

Correspondence to: Dr Hiroyuki Yoshidome, Department of General Surgery, Chiba University Graduate School of Medicine, 1-8-1 Inohana, Chuo-ku, Chiba 260-8670, Japan

E-mail: h-yoshidome@faculty.chiba-u.jp

${ }^{*}$ Contributed equally

Key words: colorectal metastases, hepatectomy, prognosis, angiogenesis for hepatic recurrence after hepatic resection for colorectal liver metastases.

\section{Introduction}

Colorectal carcinoma is among the most frequent malignant diseases worldwide, and is one of the leading causes of cancer related deaths. A significant number of patients with colorectal carcinoma who undergo curative resection unfortunately develop distant metastasis leading to a poor survival (1). The most common distant metastasis of colorectal carcinoma is to the liver, which is often associated with a poor prognosis $(2,3)$. Hepatic resection is a potentially curative therapy for colorectal liver metastases. However, recurrence (both intrahepatic and extrahepatic) occurs in approximately $60-70 \%$ of the patients after hepatectomy, suggesting that patients with colorectal liver metastasis would not always benefit from hepatectomy. Previous reports have mentioned that the timing of liver metastasis, the number of liver metastases, the presence of lymph node metastasis of the primary tumor, the presence of hilar lymph node metastasis, and the absence of fibrous capsule of the metastatic tumor are prognostic factors for survival (4-9). However, the prognostic factors for survival which are obtained from the resected specimens and the mechanism of tumor progression of the metastases have not been well elucidated.

Interaction between tumor and stromal cells may influence the tumor progression. Tumor associated macrophages which are major components of stroma have been reported to have certain roles in tumor progression (10). Monocyte chemoattractant protein-1 (MCP-1/CCL2) is a member of the $\mathrm{C}-\mathrm{C}$ chemokines that accumulate and influence macrophages/monocytes and lymphocytes which are involved in tumor progression and metastasis (11). The expression of MCP-1 has been reported in several tumor types including human melanoma, ovarian cancer, and esophageal cancer (12-14). However, only a few studies demonstrating MCP-1 expression and its biological role in colorectal carcinoma are available to date, and there are no studies investigating the correlation between survival and MCP-1 expression of colorectal liver metastasis (15-17). Although colorectal liver metastasis is considered to be a hypovascular lesion, it appears that the periphery of the metastatic lesion retains rich vascularity (18). Moreover, the efficacy of anti-vascular endothelial growth factor (VEGF) monoclonal antibody 
(bevacizumab) for unresectable or metastatic colorectal carcinoma (19) implies that angiogenesis may play a role in tumor progression in patients with colorectal liver metastasis. MCP-1 is a potent angiogenic factor in several carcinomas $(20,21)$. MCP-1 expression in breast cancer tissue has been reported to closely correlate with the concentrations of thymidine phosphorylase and VEGF, which are major components of angiogenic factors (22). We hypothesized that MCP-1 may play a role in tumor progression of colorectal liver metastases.

The objective of this study was to examine the expression of MCP-1 in colorectal liver metastasis and to determine whether MCP-1 influences tumor progression of colorectal liver metastasis by angiogenesis which is associated with survival.

\section{Patients and methods}

Patients and tissue samples. A total of 87 Japanese patients with colorectal liver metastases were selected from those who underwent curative hepatic resection (R0) at the Department of General Surgery at Chiba University (Chiba, Japan) between 1999 and 2006. Written informed consent was obtained from all the patients in accord with the ethical standards of the Helsinki Declaration of 1975. The medical records of these patients were retrospectively reviewed. In this study, synchronous metastases were defined as metastases diagnosed before colorectal surgery or at the time of surgery. The patients were followed for survival until death or June 30, 2008. The median follow-up time was 30 months (range 8-115 months). The clinicopathological characteristics of these patients are shown in Table I. In a separate experiment, two patients having high grade adenoma and three in each group having colorectal cancer of stage 0 (TNM classification), stage III, and stage IV were selected.

$H \& E$ staining and immunohistochemistry. Tissue sections (4- $\mu \mathrm{m}$ thick) were prepared from formalin-fixed paraffinembedded blocks and stained with H\&E solution. Immunohistochemistry was done using either CSA II kit or EnVision ${ }^{+}$ kit (Dako Cytomation, Carpinteria, CA) according to the manufacturer's instructions. The microwave antigen retrieval technique was used in all the cases. The series and dilutions used in the present study were as follows: a) anti-human MCP-1/CCL2 mouse monoclonal antibody (R\&D Systems), 1:50; b) anti-human CD34 mouse monoclonal antibody (Code NP036, Dako Cytomation), ready-to-use; c) anti-CD68 mouse monoclonal antibody (KP-1, Dako Cytomation), $1: 100 ;$ d) anti-Angiopoietin-2 rabbit polyclonal antibody (sc-20718, Santa Cruz Biotechnology, Santa Cruz, CA), 1:50. Appropriate positive controls known to contain the antigens in question were processed simultaneously. Negative controls were performed without the primary antibody. The tissue sections were washed in water, counterstained with Mayer's hematoxylin. The immunohistochemical analyses were done in duplicate.

Evaluation of MCP-1 and angiopoietin-2. Immunoreactivity was evaluated in a semiquantitative way assessing both staining intensity and percentage of positive cells. The expression of
Table I. MCP-1 expression and patient characteristics.

\begin{tabular}{|c|c|c|c|}
\hline \multirow[b]{2}{*}{$\begin{array}{l}\text { Clinicopathological } \\
\text { characteristics }\end{array}$} & \multicolumn{2}{|c|}{$\begin{array}{l}\text { MCP-1 expression } \\
\text { (no. of patients) }\end{array}$} & \multirow[b]{2}{*}{ P-value ${ }^{a}$} \\
\hline & $\begin{array}{l}\text { High } \\
(n=49)\end{array}$ & $\begin{array}{l}\text { Low } \\
(\mathrm{n}=38)\end{array}$ & \\
\hline Age $(\geq 60)$ & 30 & 18 & 0.284 \\
\hline Gender (man) & 29 & 26 & 0.508 \\
\hline $\begin{array}{l}\text { Lymph node metastasis } \\
\text { of the primary lesion }(+)\end{array}$ & 33 & 24 & 0.857 \\
\hline $\begin{array}{l}\text { Time of metastasis } \\
\text { (synchronous) }\end{array}$ & 23 & 15 & 0.632 \\
\hline Tumor size $(\geq 50 \mathrm{~mm})$ & 13 & 4 & 0.111 \\
\hline Fibrous capsule (+) & 11 & 4 & 0.240 \\
\hline Number of tumors $(\geq 2)$ & 40 & 22 & 0.029 \\
\hline $\begin{array}{l}\text { Primary lesion site } \\
\text { (colon) }\end{array}$ & 27 & 22 & 0.966 \\
\hline $\begin{array}{l}\text { Degree of differentiation } \\
\text { (well or moderately) }\end{array}$ & 46 & 35 & $1.00^{\mathrm{b}}$ \\
\hline
\end{tabular}

${ }^{\text {a }} \mathrm{P}$-value was determined by the $\chi^{2}$ test. ${ }^{\text {b }} \mathrm{P}$-value was determined by the Fisher's exact test.

MCP-1 and Angiopoietin-2 was determined as follows: 0, no staining; 1, occasional weak staining; 2 , moderate staining; 3 , intense staining. To qualify for scores 2 and 3 (i.e., high expression), positive staining of $>10 \%$ of tumor cells was required. The MCP-1 and Angiopoietin-2 expression was divided into two groups, the low expression group (scores 0 and 1) and the high expression group (scores 2 and 3). For the evaluation, a high-power field (x200) of 10 random areas (within the tumor) was selected. The evaluation of the immunohistochemistry was done by two investigators, T. Shida and Y. Nakatani who were not aware of the clinicopathological features and prognosis of the patients. When there was a split decision, reevaluation was done for consensus. The expressions of these molecules were evaluated both in the tumor site as well as in the invasion front of the tumor.

Evaluation of microvessel density (MVD). MVD was assessed by immunohistochemistry of CD34. The tissue sections were scanned by light microscopy at a low power field (x40) and the vessel count in areas with the highest number of capillaries was assessed at a high power field (x200). The average counts of 5 fields were determined.

Evaluation of macrophages. Macrophage was assessed by immunohistochemistry of CD68. The tissue sections were 

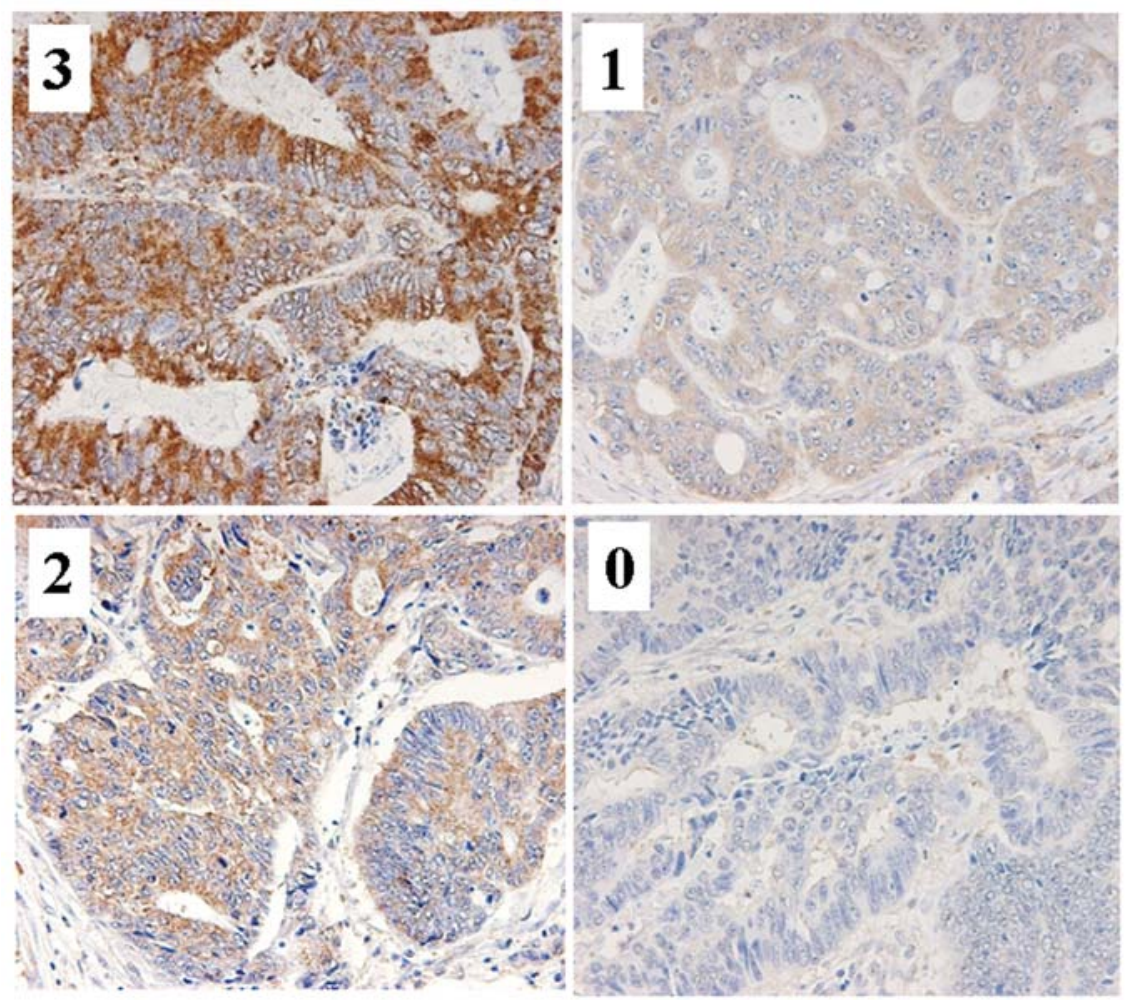

Figure 1. MCP-1 expression of the hepatic metastasis by immunohistochemistry was determined as follows: 0 , no staining; 1 , occasional weak staining; 2 , moderate staining; 3, intense staining. For the scores 2 and 3, positive expression of $>10 \%$ of tumor cells was necessary. MCP-1 was divided into two groups such as low MCP-1 expression group (scores 0 and 1) and high MCP-1 expression group (scores 2 and 3). Original magnification x200.

scanned by light microscopy at a low power field (x40) and areas with a high density of CD68 positive cells were selected. CD68 positive cells were counted at a high power field (x200) and the average counts of 5 fields were determined. According to the average number of CD68 positive cells per field (x200), the staining scores of CD68 were determined as follows: 0, 0-50 CD68 positive cells; 1, 51-100 CD68 positive cells; 2, 101-200 CD68 positive cells; 3 , $\geq 201$ CD68 positive cells

Analysis of $m R N A$ by reverse-transcription polymerase chain reaction $(R T-P C R)$. Extraction of total RNA from the cell lines was performed using RNeasy Mini kit (Qiagen, Tokyo, Japan) according to the manufacturer's instructions. Colorectal cancer cell lines were HT29, WiDr, SW48, and COLO201 (American Type Culture Collection, Manassas, VA). HT29 and WiDr are reported to have high metastatic potency (23). SW48 and COLO201 are reported to have low metastatic potency (23). Expression of MCP-1 was examined by reverse transcriptase-mediated polymerase chain reaction (RT-PCR) with the following primers: MCP-1 forward (CTC ATA GCA GCC ACC TTC AT), MCP-1 reverse (GCT TTT CCT CTT GAA CCA CA) to give a 495-bp product. GAPDH forward (TAC ACT GAG CAC CAG GTG GTC), GAPDH reverse (CCT GTT GCT GTA GCC AAA TTC G). For internal control, GAPDH cDNA was amplified. RT-PCR amplification was performed using AmpliTaq Gold PCR Master Mix (PE Applied Biosystems, Foster City, CA) and PCR thermocycler. After $10 \mathrm{~min}$ of initial melting at $95^{\circ} \mathrm{C}$, the mixture was amplified for a total of 35 cycles, using a
3 -step cycle process that began with melting at $95^{\circ} \mathrm{C}$ for $60 \mathrm{sec}$, annealing at $55^{\circ} \mathrm{C}$ for $60 \mathrm{sec}$, followed by extension at $72^{\circ} \mathrm{C}$ for $90 \mathrm{sec}$. The final cycle was followed by $12 \mathrm{~min}$ of soaking at $72^{\circ} \mathrm{C}$. Seven microliters of each RT-PCR reaction was electrophoresed in a $2.0 \%$ agarose gel and stained with ethidium bromide. RT-PCR was performed in a duplicate.

Statistical analysis. Data were compared using the MannWhitney U test or the Rank sum test. The associations between discrete variables were assessed using the $\chi^{2}$ or Fisher's exact test. The Kaplan-Meier method was used to estimate recurrence or death, and the log-rank test was used to examine statistical significance. Multivariate analysis was determined by Cox proportional hazards model. The data were analyzed by using the SigmaStat 3.0 and SPSS 11.5 software program. All data were expressed as the mean \pm $\mathrm{SD}$. The results were considered significant when $\mathrm{p}<0.05$ was obtained.

\section{Results}

MCP-1 expression of the metastatic liver tumor by immunohistochemistry. To determine whether the MCP-1 expression of the metastatic liver tumor was increased in patients with colorectal liver metastases, immunohistochemistry of MCP-1 was performed (Fig. 1). Of the 87 patients examined, 49 patients $(56.3 \%)$ showed high expression of MCP-1 (equivalent to scores $2-3)$. The remaining 38 patients $(43.7 \%)$ showed low expression of MCP-1 (equivalent to scores $0-1$ ). The staining was observed in the cytoplasm of the tumor cells. 
Table II. The correlation of MCP-1 expression with the expression of CD68 and Angiopoietin-2.

\begin{tabular}{llll}
\hline & \multicolumn{2}{c}{$\begin{array}{c}\text { MCP-1 expression } \\
(\text { no. of patients })\end{array}$} & \\
\cline { 2 - 3 } & High (n=49) & Low (n=38) & P-value \\
\hline Staining score of CD68 & $1.69 \pm 0.82$ & $1.71 \pm 0.80$ & 0.952 \\
Staining score of Ang-2 & $2.02 \pm 0.92$ & $1.50 \pm 0.97$ & 0.020 \\
\hline
\end{tabular}

Immunohistological staining scores: mean $\pm \mathrm{SD}$; ${ }^{\mathrm{a}}$ Mann-Whitney $\mathrm{U}$ test and Rank-sum test.

MCP-1 expression of the metastatic liver tumor and patient characteristics. To determine whether the MCP-1 expression of the metastatic liver tumor correlated with the clinicopathological parameters, we examined patient characteristics. MCP-1 expression of the metastatic liver tumor and clinicopathological parameters were demonstrated in Table I. The number of patients with multiple metastatic liver tumors significantly increased in the high MCP-1 expression group relative to that in the low MCP-1 expression group ( $\mathrm{p}=0.029)$, while no significant difference was found in other clinicopathological parameters between the two groups.

Relationship of MCP-1 and CD68 expression. One of the roles of MCP-1 has been reported to accumulate monocytes/ macrophages and lymphocytes including tumor-associated macrophages (11). To determine whether the MCP-1 expression of the metastatic liver tumor was correlated with the accumulation of macrophages, we examined the expression of CD68 which is the marker of macrophages (24) and determined the staining scores as defined in the methods section. The results of the immunohistochemical staining scores are listed in Table II. While the accumulation of macrophages defined by CD68 immunohistochemistry in the tumor was observed, there was no correlation between the expression of MCP-1 and CD68 (Table II).

Relationship of MCP-1, Angiopoietin-2, and MVD (CD34) expression. MCP-1 has been demonstrated to be related with angiogenesis (22). To determine whether the MCP-1 expression of the metastatic liver tumor correlated with angiogenesis, we examined the expression of CD34 and the MVD count. As the MCP-1 expression increased (scoring 2 and 3), the MVD count significantly increased in comparison to little MCP-1 expression (scoring 0, Fig. 2A). An intense staining of Angiopoietin-2 expression of the tumor was correlated with an increase in the MVD count (Fig. 2B). As shown in Table II, the expression of MCP-1 of the tumor positively correlated with Angiopoietin-2 expression $(\mathrm{p}=0.020)$.

MCP-1 expression of the primary colorectal cancer and clinical stage. In a separate experiment, to determine whether the degree of MCP-1 expression was related to the clinical
A

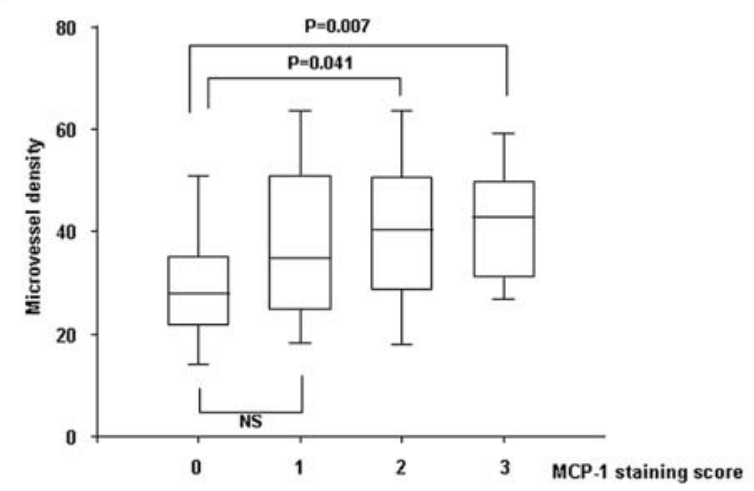

B

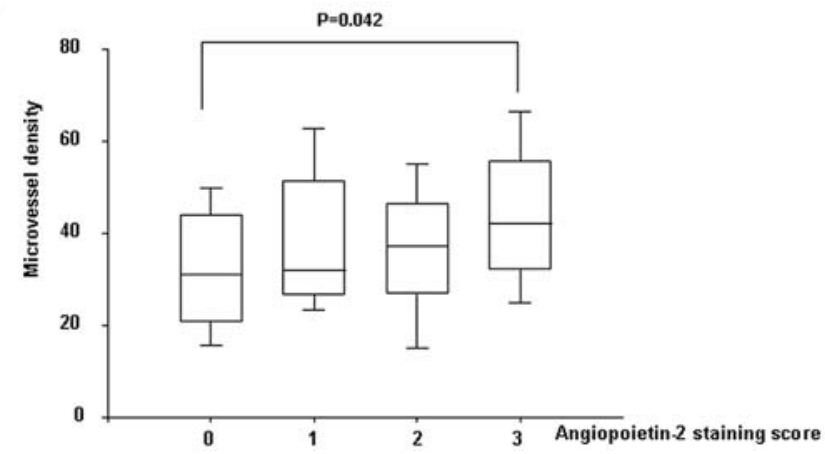

Figure 2. Microvessel density (MVD) count by immunohistochemistry for CD34 was examined according to MCP-1 (A) and Angiopoietin-2 (B) expression. Data are presented as box plots: the extent of the boxes indicate the 25 th and the 75 th percentiles, the lines inside the boxes mark 50th percentile (median) values. The capped bars indicate the 5 th and 95 th percentiles. A $\mathrm{p}<0.05$ was considered to be significant.

stage, we examined the MCP-1 expression by immunohistochemistry for the primary colorectal tumor stratified by the adenoma high grade or primary colorectal cancer defined by UICC classification. Little MCP-1 expression was observed in adenoma high grade $(n=2)$ and stage 0 colorectal cancer (n=3, Fig. 3A and B). As the clinical stage increased (III and IV), moderate MCP-1 expression was found in stage III colorectal cancer $(n=3)$ and significantly intense MCP-1 expression was found in stage IV colorectal cancer $(n=3)$ in comparison to the early stage colorectal cancer (Fig. 3C and D).

MCP-1 mRNA expression of colon cancer cell lines. To determine the MCP-1 expression of colon cancer cell lines, which are stratified by metastasis potency, RT-PCR was performed. MCP-1 mRNA expression was increased in cancer cell lines which have high metastasis potency such as HT29. On the contrary, MCP-1 mRNA expression was relatively decreased in cancer cell line with low metastasis potency such as SW48 and COLO201 (Fig. 4).

$M C P-1$ expression and survival. To determine whether the MCP-1 expression of the metastatic liver tumor was related to hepatic recurrence or overall survival after hepatic resection, we examined hepatic disease-free or overall survival with the Kaplan-Meier method. Most of hepatic recurrence was observed within 2 years after hepatectomy. The hepatic 

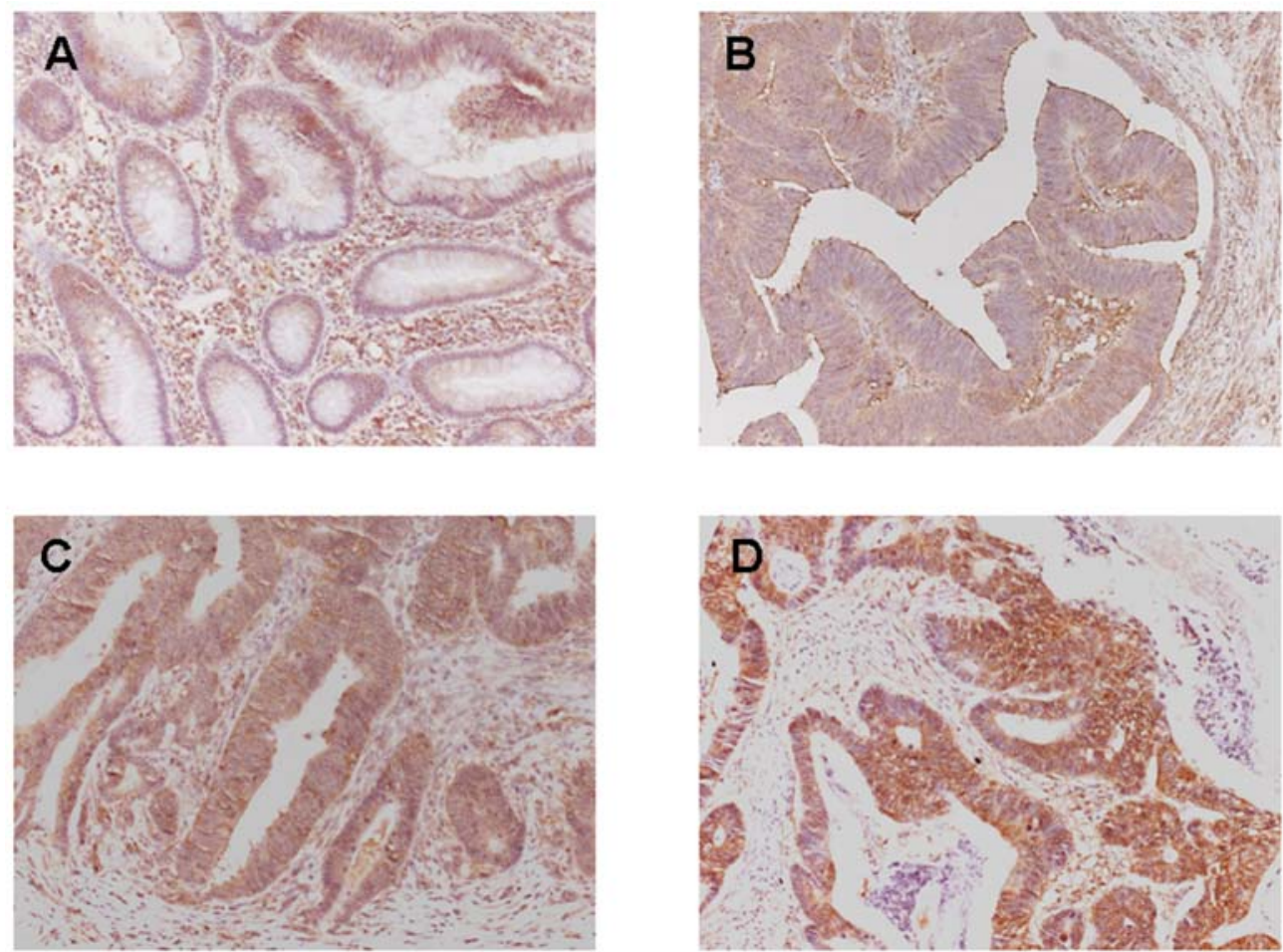

Figure 3. MCP-1 expression of the primary colorectal cancer by immunohistochemistry was determined as follows: (A), high grade adenoma; (B), stage 0 colorectal cancer; (C), stage III colorectal cancer; (D), stage IV colorectal cancer. Clinical stage was identified by TNM classification. Original magnification $\mathrm{x} 200$.

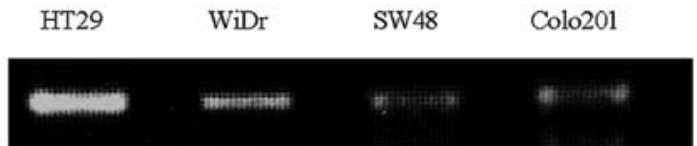

GAPDH

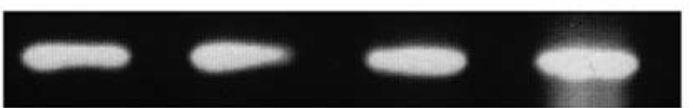

Figure 4. mRNA expression of MCP-1 of the colon cancer cell lines by RT-PCR. MCP-1 mRNA expression was increased in cancer cell lines with high metastatic potency, HT29 and WiDr have a high metastatic potency and SW48 and COLO201 have a low metastatic potency.

disease-free survival was significantly lower in patients in the high MCP-1 expression group relative to patients in the low MCP-1 expression group ( $p=0.0014$, Fig. 5A). Although the follow-up periods may not be long enough, the overall survival in patients with high MCP-1 expression group was worse compared with low MCP-1 expression group ( $\mathrm{p}=0.0407$, Fig. 5B).

To determine whether the MCP-1 expression was a significant prognostic factor for hepatic disease-free survival after hepatic resection, we performed both uni- and multivariate analyses. The univariate analysis demonstrated that the timing of hepatic metastases, tumor size, number of metastases, and MCP-1 expression of the tumor were significant prognostic factors (Table III). The multivariate analysis showed that MCP-1 expression and the timing of hepatic metastases were significant independent prognostic factors for hepatic disease-free survival in patients with colorectal liver metastases (Table III).

\section{Discussion}

This study revealed that MCP-1 is aberrantly expressed in subsets of metastatic colorectal liver tumors, and is related with a poor prognosis, especially with regard to intrahepatic recurrence. The expression of MCP-1 has been reported in several malignancies in relation to tumor progression, but only limited data are currently available with regard to colorectal carcinoma (12-17). Furthermore, no study has been performed on a large number of colorectal metastatic liver tumors with regard to the correlation between MCP-1 expression and prognosis.

The role for MCP-1 in tumor progression remains controversial $(20,25,26)$. In the current study, the MCP-1 expression of primary colorectal cancer increased as the clinical stage (UICC classification) advanced, suggesting that MCP-1 appears to be related to metastasis potency of colorectal cancer. Along with this finding, MCP-1 mRNA expression was relatively increased in cancer cell lines which have high metastasis potency in comparison to those with low metastasis potency. Thus, these findings suggested that MCP-1 may play a certain role in tumor progression and metastasis.

One of the roles of MCP-1 is to recruit macrophages (11). However, in the present study, no positive relationship was observed between the degree of MCP-1 expression and accumulation of tumor-associated macrophages. Although the expression of tumor-associated macrophages is reported to be associated with a poor prognosis in several malignancies (i.e., breast cancer, bladder cancer, and prostate cancer), as for colorectal carcinoma, the expression of tumorassociated macrophages has been reported with a favorable 


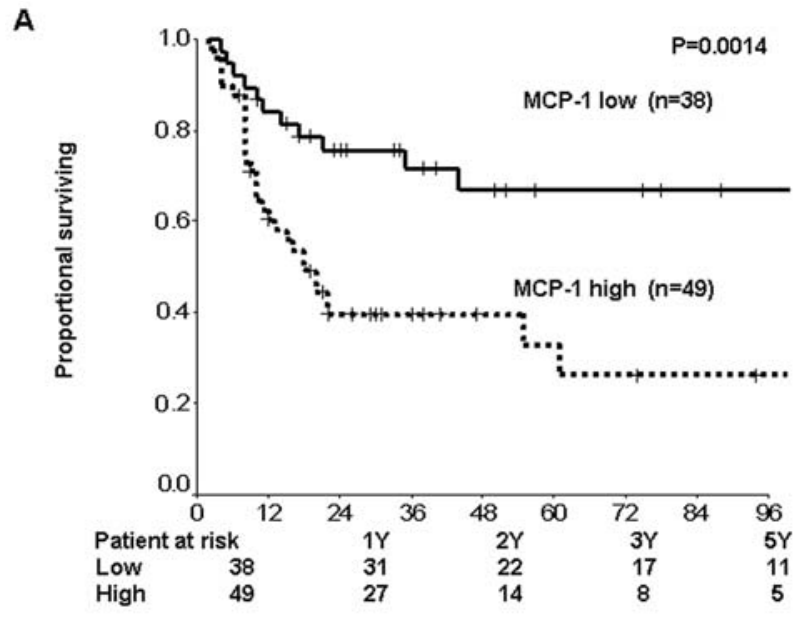

B

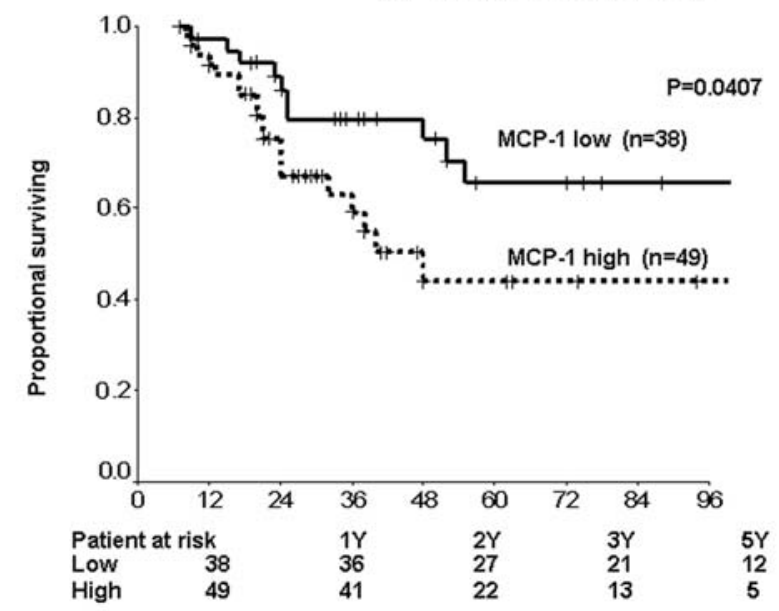

Figure 5. (A), The hepatic disease-free survival rate stratified by high MCP-1 expression group $(n=49)$ or low MCP- 1 expression group $(n=38)$. Hepatic disease-free survival of patients after hepatectomy was calculated by the Kaplan-Meier method. A comparison was performed using the log-rank test. High MCP-1 expression was significantly associated with shorter diseasefree survival $(\mathrm{p}=0.0140)$. (B) The overall survival rate stratified by high MCP-1 expression group ( $\mathrm{n}=49$ ) or low MCP-1 expression group ( $\mathrm{n}=38)$. The overall survival of patients after hepatectomy was calculated by the Kaplan-Meier method. The comparison was performed using the log-rank test.

prognosis $(24,27,28)$. Therefore, the observation concerning tumor-associated macrophages and the prognosis in various cancers seems to be still a matter of debate. The accumulation of tumor-associated macrophages may promote tumor progression by altering the balance of the macrophages, however it may be a reflection of the increased inflammatory activity within cancer patients. The present data suggest that there may be other chemoattractants that would influence the accumulation of tumor-associated macrophages in colorectal liver metastasis. The relationship of MCP-1 expression and tumor-associated macrophages in colorectal liver metastases thus needs further investigation.

Another role for MCP-1 is a potent angiogenic factor $(20,21,29)$. The concentration of MCP-1 in breast cancer tissue has been reported to closely correlate with the concentrations of thymidine phosphorylase and VEGF, which are major components of angiogenic factors (22). In the current study, a significant correlation was observed between the MCP-1 expression and microvessel density defined by CD34 immuno-
Table III. Univariate and multivariate analysis of predictive factors for hepatic recurrence.

\begin{tabular}{lll}
\hline Factors & 5-year (MST, month) & P-value \\
\hline $\begin{array}{l}\text { Time of metastases } \\
\text { Synchronous ( } \mathrm{n}=38)\end{array}$ & $29 \%(18)$ & 0.0030 \\
Metachronous ( $\mathrm{n}=49)$ & $65 \%$ & \\
Tumor size & & \\
$<50$ mm ( $=70)$ & $57 \%$ & 0.0016 \\
$\geq 50$ mm (n=17) & $13 \%(14)$ & \\
Number of metastases & & \\
$\leq 4(\mathrm{n}=71)$ & $53 \%$ & \\
$>5$ ( $\mathrm{n}=16)$ & $25 \%(15)$ & \\
MCP-1 expression & & \\
Low (n=38) & $67 \%$ & \\
High (n=49) & $32 \%(18)$ & \\
\hline
\end{tabular}

${ }^{\mathrm{a}}$ Log-rank test.

\begin{tabular}{lcccc}
\hline & \multicolumn{4}{c}{$\begin{array}{c}95 \% \text { confidence } \\
\text { Fntervals }\end{array}$} \\
\cline { 3 - 4 } & $\begin{array}{c}\text { Relative } \\
\text { risk }\end{array}$ & Lower & Upper & P-value \\
\hline $\begin{array}{l}\text { Time of metastases } \\
\text { (synchronous:meta- } \\
\text { chronous) }\end{array}$ & 2.24 & 1.14 & 4.42 & 0.020 \\
$\begin{array}{l}\text { Tumor size } \\
(\geq 50:<50)\end{array}$ & 1.94 & 0.76 & 4.96 & 0.166 \\
$\begin{array}{l}\text { Number of metastases } \\
(\leq 4:>5)\end{array}$ & 0.94 & 0.36 & 2.48 & 0.903 \\
$\begin{array}{l}\text { MCP-1 expression } \\
\text { (high:low) }\end{array}$ & 2.96 & 1.45 & 6.05 & 0.003 \\
\hline
\end{tabular}

${ }^{\mathrm{b}}$ Cox proportional hazards model.

histochemistry in colorectal liver metastatic tumor. Consistent with this finding, MCP-1 has been demonstrated to be associated with tumor vessel density in human esophageal cancer (20). Moreover, the MCP-1 expression was positively related with the Angiopoietin-2 expression which is a representative angiogenic factor. Angiopoietin-2 is one of the potent and crucial factors for angiogenesis, and its role has been previously studied in human hepatocellular carcinoma (30). Concerning colorectal carcinoma, it has been revealed that Angiopoietin-2 contributes to angiogenesis and tumor growth leading to a shorter survival $(31,32)$. These findings may support the present study that MCP-1, at least in part, may contribute to angiogenesis and be related to a poor survival in patients with colorectal liver metastasis. Thus, it 
seems likely that the MCP-1 expression plays a role in angiogenesis in patients with colorectal liver metastasis.

Regarding the clinicopathological features, the high MCP-1 expression was associated with multiple colorectal metastatic liver tumors, suggesting that intrahepatic spread might be increased in patients with high MCP-1 expression of the tumors. Moreover, MCP-1 was a significant independent prognostic factor for the hepatic disease-free survival in comparison to other previously reported clinical factors (4-9). Thus, the MCP-1 expression of the metastatic liver tumor may be an indicator for the prediction of remnant liver recurrence. In overall survival, the MCP-1 expression also reached statistical difference, although the significance was less than that of hepatic disease-free survival. This may be explained by the fact that extrahepatic or other peritoneal recurrence was occurred in some patients with colorectal liver metastases after hepatectomy. Taken together, in patients with colorectal liver metastasis, it seems likely that MCP-1 plays crucial roles in not only angiogenic promotion but in biologically related aggressive behavior as well, and the score of MCP-1 expression of the metastatic liver tumors may be useful in predicting the clinical outcome of patients with colorectal liver metastases after hepatectomy.

In conclusion, the high expression of MCP-1 in tumors of colorectal metastases may cause tumor progression by angiogenesis and be useful in predicting hepatic recurrence and survival after hepatectomy. Further and a larger number of studies are needed to evaluate the more precise mechanism of MCP-1 in patients with colorectal liver metastasis.

\section{Acknowledgements}

This work was supported in part by grants from the Japan Society for the Promotion of Science (grant no. 19591577).

\section{References}

1. Rosen M, Chan L, Beart RW Jr, Vukasin P and Anthone G: Follow-up of colorectal cancer: a meta-analysis. Dis Colon Rectum 41: 1116-1126, 1998 .

2. Nordlinger B, Guiguet M, Vaillant JC, Balladur K, Boudjema P Bachellier $\mathrm{P}$ and Jaeck D: Surgical resection of colorectal carcinoma metastases to the liver. A prognostic scoring system to improve case selection, based on 1568 patients. Association Française de Chirurgie. Cancer 77: 1254-1262, 1996.

3. Fong Y, Cohen AM, Fortner JG, Enker WE, Turnbull AD Coit DG, Marrero AM, Prasad M, Blumgart LH and Brennan MF: Liver resection for colorectal metastases. J Clin Oncol 15 : 938-946, 1997

4. Minagawa M, Makuuchi M, Torzilli G, Takayama T, Kawasaki S, Kosuge T, Yamamoto J and Imamura H: Extension of the frontiers of surgical indications in the treatment of liver metastasis from colorectal cancer: long-term results. Ann Surg 231: 487-499, 2000

5. Ambiru S, Miyazaki M, Isono T, Ito H, Nakagawa K, Shimizu H, Kusashio K, Furuya S and Nakajima N: Hepatic resection for colorectal metastases: analysis of prognostic factors. Dis Colon Rectum 42: 632-639, 1999.

6. Yasui K, Hirai T, Kato T, Torii A, Uesaka K, Morimoto T, Kodera Y, Yamamura Y, Kito T and Hamajima N: A new macroscopic classification predicts prognosis for patients with liver metastases from colorectal cancer. Ann Surg 226: 582-586, 1997.

7. Fong Y, Fortner J, Sun RL, Brennan MF and Blumgart LH: Clinical score for predicting recurrence after hepatic resection for metastatic colorectal cancer: analysis of 1001 consecutive cases. Ann Surg 230: 309-318, 1999.
8. Scheele J, Stang R, Altendorf-Hofmann A and Paul M: Resection of colorectal liver metastases. World J Surg 19: 59-71, 1995.

9. Rogers MS and McCall JL: Surgery for colorectal liver metastases with hepatic lymph node involvement: a systematic review. Br J Surg 87: 1142-1155, 2000.

10. Mantovani A, Bottazzi B, Colotta F, Sozzani S and Ruco L: The origin and function of tumor-associated macrophages. Immunol Today 13: 265-270, 1992.

11. Oppenheim JJ, Zachariae CO, Mukaida N and Matsushima K: Properties of the novel proinflammatory supergene 'intercrine' cytokine family. Annu Rev Immunol 9: 617-648, 1991.

12. Graves DT, Barnhill R, Galanopoulos T and Antoniades HN: Expression of monocyte chemotactic protein-1 in human melanoma in vivo. Am J Pathol 140: 9-14, 1992.

13. Negus RP, Stamp GW, Relf MG, Burke F, Malik ST, Bernasconi S, Allavena P, Sozzani S, Mantovani A and Balkwill FR: The detection and localization of monocyte chemoattractant protein-1 (MCP-1) in human ovarian cancer. J Clin Invest 95: 2391-2396, 1995.

14. Koide N, Nishio A, Sato T, Sugiyama A and Miyagawa S: Significance of macrophage chemoattractant protein-1 expression and macrophage infiltration in squamous cell carcinoma of the esophagus. Am J Gastroenterol 99: 1667-1674, 2004.

15. Tanaka S, Tatsuguchi A, Futagami S, Gudis K, Wada K, Seo T, Mitsui K, Yonezawa M, Nagata K, Fujimori S, Tsukui T, Kishida $\mathrm{T}$ and Sakamoto $\mathrm{C}$ : Monocyte chemoattractant protein 1 and macrophage cyclooxygenase 2 expression in colonic adenoma. Gut 55: 54-61, 2006.

16. Bailey C, Negus R, Morris A, Ziprin P, Goldin R, Allavena P, Peck D and Darzi A: Chemokine expression is associated with the accumulation of tumour associated macrophages (TAMs) and progression in human colorectal cancer. Clin Exp Metastasis 24: 121-130, 2007.

17. Cheadle EJ, Riyad K, Subar D, Rothwell DG, Ashton G, Batha H, Sherlock DJ, Hawkins RE and Gilham DE: Eotaxin-2 and colorectal cancer: a potential target for immune therapy. Clin Cancer Res 13: 5719-5728, 2007.

18. Murakami T, Kim T, Takamura M, Hori M, Takahashi S Federle MP, Tsuda K, Osuga K, Kawata S, Nakamura H and Kudo M: Hypervascular hepatocellular carcinoma: detection with double arterial phase multi-detector row helical CT. Radiology 218: 763-767, 2001.

19. Saltz LB, Clarke S, Díaz-Rubio E, Scheithauer W, Figer A, Wong R, Koski S, Lichinitser M, Yang TS, Rivera F, Couture F, Sirzén F and Cassidy J: Bevacizumab in combination with oxaliplatin-based chemotherapy as first-line therapy in metastatic colorectal cancer: a randomized phase III study. J Clin Oncol 26: 2013-2019, 2008.

20. Ohta M, Kitadai Y, Tanaka S, Yoshihara M, Yasui W, Mukaida N, Haruma K and Chayama K: Monocyte chemoattractant protein-1 expression correlates with macrophage infiltration and tumor vascularity in human gastric carcinomas. Int J Oncol 22: 773-778, 2003.

21. Leek RD, Lewis CE, Whitehouse R, Greenall M, Clarke J and Harris AL: Association of macrophage infiltration with angiogenesis and prognosis in invasive breast carcinoma. Cancer Res 56: 4625-4629, 1996.

22. Ueno T, Toi M, Saji H, Muta M, Bando H, Kuroi K, Koike M, Inadera $\mathrm{H}$ and Matsushima K: Significance of macrophage chemoattractant protein-1 in macrophage recruitment, angiogenesis, and survival in human breast cancer. Clin Cancer Res 6: 3282-3289, 2000.

23. Hamada K, Monnai M, Kawai K, Nishime C, Kito C, Miyazaki N, Ohnishi Y, Nakamura $M$ and Suemizu H: Liver metastasis models of colon cancer for evaluation of drug efficacy using NOD/Shi-scid IL2R $\gamma$ null (NOG) mice. Int J Oncol 32: 153-159, 2008.

24. Khorana AA, Ryan CK, Cox C, Eberly S and Sahasrabudhe DM: Vasucular endothelial growth factor, CD68, and epidermal growth factor receptor expression and survival in patients with stage II and stage III colon carcinoma: a role for the host response in prognosis. Cancer 97: 960-968, 2003.

25. Nakashima E, Mukaida N, Kubota Y, Kuno K, Yasumoto K, Ichimura F, Nakanishi I, Miyasaka M and Matsuhima K: Human MCAF gene transfer enhances the metastatic capacity of a mouse cachectic adenocarcinoma cell line in vivo. Pharmacol Res 12: 1598-1604, 1995.

26. Rollins BJ and Sunday ME: Suppression of tumor formation in vivo by expression of the JE gene in malignant cells. Mol Cell Biol 11: 3125-3131, 1991. 
27. Lissbrant IF, Stattin P, Wikstrom P, Damber JE, Egevad L and Bergh A: Tumor associated macrophages in human prostate cancer: relation to clinicopathological variables and survival. Int J Oncol 17: 445-451, 2000.

28. Hanada T, Nakagawa M, Emoto A, Nomura T, Nasu N and Nomura Y: Prognostic value of tumor-associated macrophage count in human bladder cancer. Int J Urol 7: 263-269, 2000.

29. Niu J, Azfer A, Zhelyabovska O, Fatma S and Kolattukudy PE: Monocyte chemotactic protein (MCP)-1 promotes angiogenesis via a novel transcription factor, MCP-1-induced protein (MCPIP). J Biol Chem 283: 14542-14551, 2008.

30. Mitsuhashi N, Shimizu H, Ohtsuka M, Wakabayashi Y, Ito H, Kimura F, Yoshidome H, Kato A, Nukui Y and Miyazaki M: Angiopoietins and Tie-2 expression in angiogenesis and proliferation of human hepatocellular carcinoma. Hepatology 37: 1105-1113, 2003.
31. Ogawa M, Yamamoto H, Nagano H, Miyake Y, Sugita Y, Hata T, Kim BN, Ngan CY, Damdinsuren B, Ikenaga M, Ikeda M, Ohue M, Nakamori S, Sekimoto M, Sakon M, Matsuura N and Monden M: Hepatic expression of Ang-2 RNA in metastatic colorectal cancer. Hepatology 39: 528-539, 2004. 32. Ahmad SA, Liu W, Jung YD, Fan F, Wilson M, Reinmuth N, Shaheen RM, Bucana CD and Ellis LM: The effects of angiopoietin-1 and -2 on tumor growth and angiogenesis in human colon cancer. Cancer Res 61: 1255-1259, 2001. 